\title{
The Neural-based Segmentation of Cursive Words using Enhanced Heuristics
}

\author{
Chun Ki Cheng and Michael Blumenstein \\ School of Information and Communication Technology, Griffith University-Gold Coast Campus \\ PMB 50, Gold Coast Mail Centre, QLD 9726, Australia \\ m.blumenstein@griffith.edu.au
}

\begin{abstract}
This paper presents an Enhanced Heuristic Segmenter (EHS) and an improved neural-based segmentation technique for segmenting cursive words and validating prospective segmentation points respectively. The EHS employs two new features, ligature detection and a neural assistant, to locate prospective segmentation points. The improved neural-based segmentation technique can then be used to examine the prospective segmentation points by fusion of confidence values obtained from left and centre character recognition outputs in addition to the segmentation point validation (SPV) output. The improved neural-based segmentation technique uses a recently proposed feature extraction technique (Modified Direction Feature) for representing the segmentation points and characters to enhance the overall segmentation process. The EHS and the neural-based segmentation technique have been implemented and tested on a benchmark database providing encouraging results.
\end{abstract}

\section{Introduction}

Off-line handwriting recognition is one of the most challenging research problems due to the different variations in handwriting [1]. The motivations in this field include the growing demand of commercial applications and the scientific progress in an age-old artificial intelligence problem.

Recent research into the recognition of isolated characters has produced some promising results [2-4]. However, in terms of segmentation-based word recognition, the recognition rate can be low for poorly segmented words. One typical approach in the literature is over-segmentation [5-7]. The purpose of 'oversegmentation' is to dissect the word into a sufficient number of components so that no merged characters remain. One of the major problems following oversegmentation is to correctly discard invalid segmentation points.

In this paper, an existing neural-based segmentation technique [8] is enhanced to validate prospective segmentation points. The existing technique first uses a Feature-based Heuristic Segmenter (FHS) [9] to oversegment the handwriting. Following this, a neural confidence-based module is used to evaluate a prospective segmentation point by obtaining a fused value from three neural confidence values: SPV, left character validation $(\mathrm{LCV})$ and center character validation $(\mathrm{CCV})$. The segmentation technique has two advantages. Firstly, it can reduce the number of missed segmentation points and hence increase the overall character/word recognition rate. Secondly, since the number of segmentation points is optimized directly following over-segmentation, it can reduce the processing time of later stages.

The enhancements to the existing segmentation technique include an Enhanced Heuristic Segmenter (EHS) that employs ligature detection and a neural assistant for obtaining better prospective segmentation points (PSPs). In addition, the neural confidence-based module is improved by using 1) a recently proposed feature extraction technique [4] for processing relevant features, 2) a single character classifier for the recognition of left characters and center characters and 3) a segmentation path detection-based character extraction technique [10].

The remainder of the paper is broken down into 4 sections. Section 2 describes the enhanced neural-based segmentation technique. Section 3 provides experimental results, followed by discussion in Section 4. Finally, conclusions are drawn in Section 5.

\section{Enhanced Segmentation Technique}

This section presents some enhancements to the neural-based segmentation technique. The new heuristic segmenter, EHS, employs two new attributes - ligature detection and a neural assistant. The first component was investigated since the former segmenter, FHS, could not effectively locate prospective segmentation points that were located under over-lapped strokes. The second feature, the neural assistant, uses a hybrid strategy that combines a character classifier and heuristic rules to oversegment the handwriting. Figure 1 shows an overview of the EHS algorithm. 


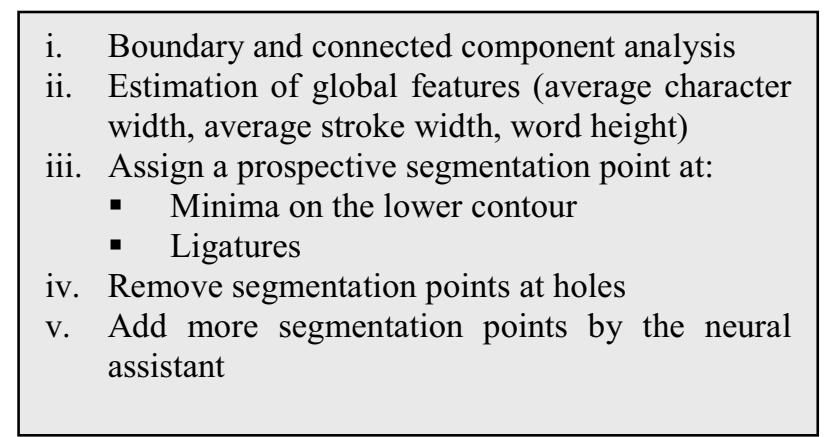

Figure 1. Overview of EHS algorithm

The improved neural confidence-based module uses a newly proposed feature extraction technique, Modified Direction Feature (MDF) for SPV, LCV and CCV. LCV and $\mathrm{CCV}$ use a single classifier for character recognition and a Segmentation Path Detection (SPD) technique is used to extract characters for the recognition process. Figure 2 illustrates an overview of the entire neural-based segmentation technique.

In the following sub-sections, further details of ligature detection, the neural assistant, MDF and SPD are provided.

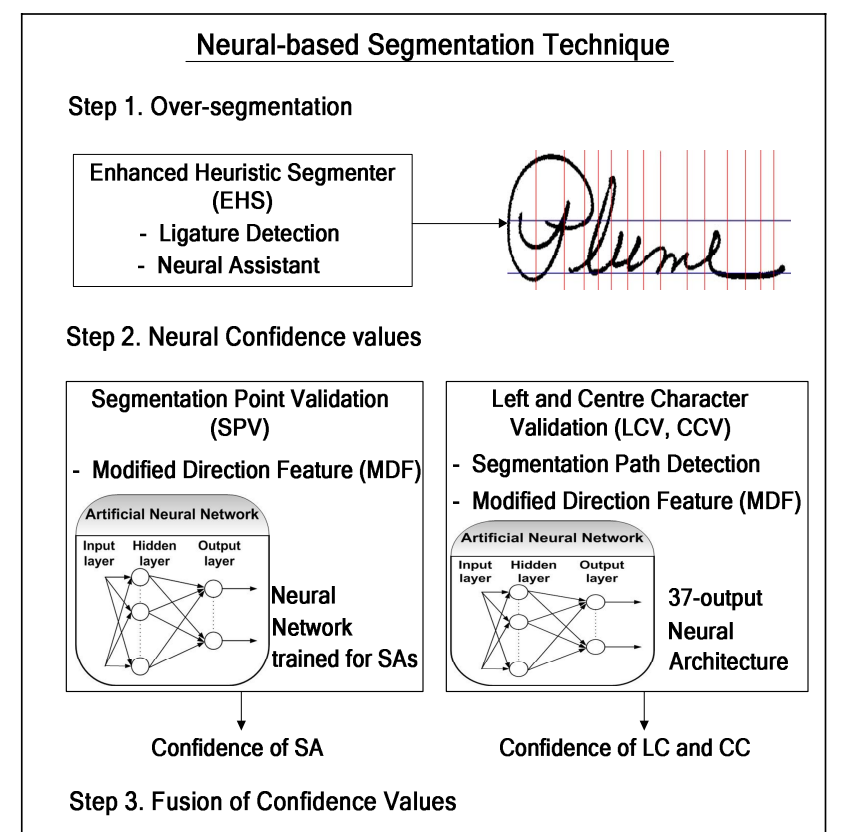

Figure 2. Overview of the improved, neuralbased segmentation technique

\subsection{Ligature Detection}

A ligature is a small stroke that is used to connect joined/cursive characters. One of the major features of a ligature is that it is usually located within the "middleregion" of handwritten words. Hence, a baseline detection technique can be used to identify this middle region.
Following this, a modified vertical histogram is generated based on the middle region of the handwriting to locate possible ligatures.

2.1.1. Baseline detection. Small strokes in a word image may extend above or below the main body of handwriting. Such letter components are called ascenders and descenders respectively. Examples of letters that contain such strokes are: ' $f$ ', ' $j$ ', ' $g$ ', ' $T$ ' etc. Hence the letters that contain ascenders or descenders may overlap parts of characters in the main body that do not contain such strokes. In order to over-segment the word image more accurately, it is necessary to remove ascenders and descenders before the actual segmentation process. In this research, the technique calculates the average vertical value of the maxima and minima on the upper and lower contours respectively. Abnormal maxima and minima are removed based on this average value. Finally, baselines are estimated by the average of the remaining maxima and minima.

2.1.2. Modified vertical histogram. The second step in the ligature detection algorithm is to analyse the middle region and to locate ligatures. One common approach is the use of vertical (density) histogram analysis. The analysis is based on the vertical distribution of foreground pixels. The histogram is drawn by a projection of the total number of foreground pixels in each column of the word image. Areas with low pixel density are then identified as possible segmentation points. Figure 3 illustrates an example vertical histogram; the vertical histogram is formed based on the middle region of the word "Top".

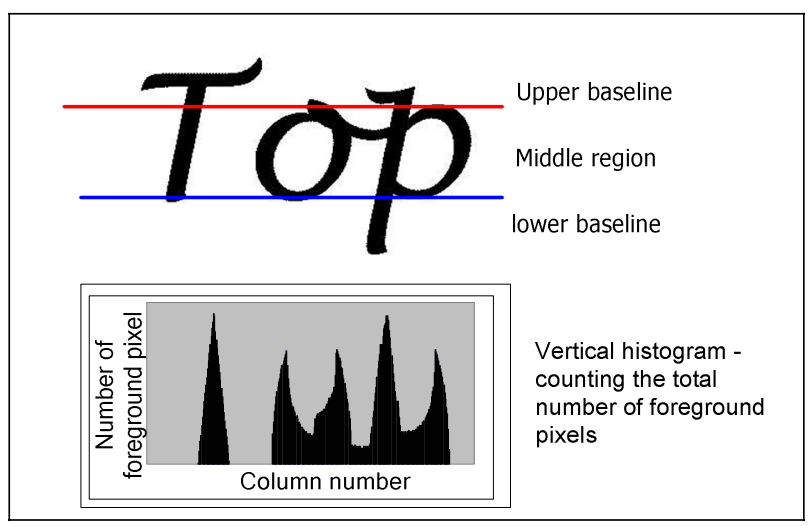

Figure 3. Vertical histogram analysis

Figure 3 illustrates that there are an excessive number of "low" density regions. This is because the vertical histogram is not adequate to distinguish the difference between "holes" and "ligatures". In this research, a modified vertical histogram was developed to improve the accuracy of locating ligatures. Figure 4 shows the modified vertical histogram of the word shown in Figure 3. 


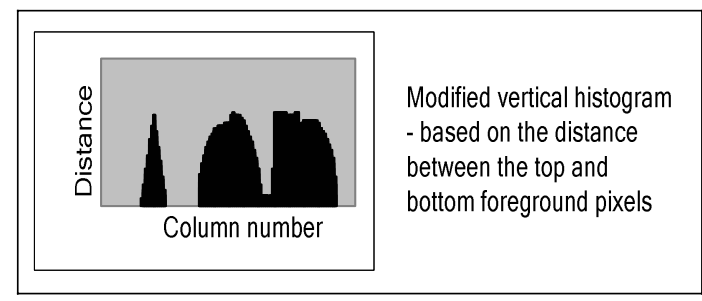

Figure 4. Modified vertical histogram analysis

The concept of the modified vertical histogram is formed by calculating the distance between the top and bottom foreground pixels for each column in a word image. As may be seen from Figure 4, the ligature region is clear and hence easy for the segmenter to detect. One weakness of the modified vertical histogram is that it is not suitable for characters with overlapped strokes. But in this research, since the overlapped strokes are removed in most cases (i.e. the modified vertical histogram is formed from the middle region), the advantage of the modified vertical histogram can then be maximized.

Ligatures are located using the modified vertical histogram and a heuristic based on the average stoke width. Regions with distance values smaller than the average stroke width are defined as ligatures.

\subsection{Neural Assistant}

The neural assistant uses a character classifier and some extra heuristics to generate additional segmentation points following regular feature-based segmentation point assignment. Regions between two successive prospective segmentation points are extracted and processed by MDF in order to obtain a confidence value. Additional segmentation points are added based on the confidence value and the distance between the two prospective segmentation points. Experimental results in [10] showed that the classifier could be effectively used to distinguish character and non-character component, and hence could provide appropriate assistance in the current step.

\subsection{Modified Direction Feature (MDF)}

Recent work has shown that the Modified Direction Feature (MDF) enhances the character recognition process and outperforms some popular feature extraction technique such as the Transition Feature (TF) [4]. This work demonstrated the superiority of MDF for describing patterns based on their contour or boundary. This prompted an investigation to determine the feasibility of employing MDF for SPV, LC and CC recognition to enhance the overall segmentation process. The details of MDF have been described in [4].

\subsection{Segmentation Path Detection (SPD)}

The SPD character extraction technique was used to "cleanly" extract characters for LCV and CCV that may have been overlapping with other characters strokes. It has been described in more detail in [10] [11].

\section{Experimental Results}

Two sets of experiments were conducted in this research. Both experiments use the improved neural confidence-based module for validating the prospective segmentation points, but different heuristic segmenters were used to over-segment the handwriting. In addition, the performance of EHS and FHS was compared.

Segmentation performance is measured based on three types of segmentation errors: over-segmentation, "missed" and "bad" metrics. Over-segmentation refers to a character that has been divided into more than three components. A "missed" error occurs when no segmentation point is found between two successive characters. The "bad" error refers to a segmentation point that could not be used to extract a character precisely.

\subsection{Handwriting Database}

The training and testing patterns for this work were obtained from handwritten words contained in the CEDAR benchmark database [12] "/train/cities/BD" and "/test/cities/BD" directories respectively.

\subsection{Neural Network Configuration}

The classifiers used in this research were feed-forward Multi-layered Perceptrons (MLPs) trained with the resilient backpropagation (BP) algorithm. For experimental purposes, the architectures were modified varying the number of inputs, outputs and hidden units.

\subsection{EHS Segmentation Performance}

Table 1 shows the segmentation performance of FHS and EHS. The results are based on the 1031 segmentation points that existed between joined characters.

Table 1: Segmentation performance of EHS and FHS (1031 segmentation points)

\begin{tabular}{|c|c|c|c|}
\hline \multirow{2}{*}{} & \multicolumn{3}{|c|}{ Segmentation Error Rates } \\
\cline { 2 - 4 } & $\begin{array}{c}\text { Over-segmented } \\
{[\%]}\end{array}$ & $\begin{array}{c}\text { Missed } \\
{[\%]}\end{array}$ & $\begin{array}{c}\text { Bad } \\
{[\%]}\end{array}$ \\
\hline FHS & 4.07 & 4.07 & 6.99 \\
\hline EHS & 2.72 & 2.42 & 4.56 \\
\hline
\end{tabular}




\subsection{Performance of the Neural-based Segmentation Technique}

The errors of the enhanced neural-based segmentation technique are calculated based on the number of correct segmentation points in word samples. The total number of segmentation points in the 317 word samples is 1718 . Only 1031 segmentation points that existed between joined characters are chosen for testing purpose. The reason for this is to test the segmenter on its ability to separate cursive character components. Table 2 shows the overall results of the enhanced neural-based segmentation technique and the existing neural-based segmentation technique using 317 testing words.

\section{Table 2: Overall results of the neural-based} segmentation technique (1031 segmentation points)

\begin{tabular}{|c|c|c|c|}
\hline & \multicolumn{3}{|c|}{ Segmentation Error Rates } \\
\cline { 2 - 4 } & $\begin{array}{c}\text { Over-segmented } \\
{[\%]}\end{array}$ & $\begin{array}{c}\text { Missed } \\
{[\%]}\end{array}$ & $\begin{array}{c}\text { Bad } \\
{[\%]}\end{array}$ \\
\hline $\begin{array}{c}\text { Existing } \\
\text { Technique }\end{array}$ & 7.08 & 2.33 & 10.86 \\
\hline $\begin{array}{c}\text { Experiment } \\
\text { 1 (FHS) }\end{array}$ & 8.73 & 0.1 & 8.63 \\
\hline $\begin{array}{c}\text { Experiment } \\
\text { 2 (EHS) }\end{array}$ & 7.37 & 0.1 & 6.79 \\
\hline
\end{tabular}

\section{Analysis and Discussion of Results}

\subsection{Analysis of EHS Over-segmentation}

The introduction of ligature detection to locate prospective segmentation points hidden by large horizontal strokes or overlapping characters proved quite successful. As may be seen from Table 1, EHS performed fairly well on the test set with only $2.42 \%$ of 'missed errors'.

Two problems were found during the inspection process. The first problem arises when segmenting very noisy characters. Since the enhanced heuristic algorithm was heavily dependent on contour analysis, heavy noise that was inherent around the handwriting could cause serious errors. One of the solutions to this problem was additional pre-processing.

The second problem that was observed related to the neural assistant. The main problem was incorrect classification. However, overall the classification rate was acceptable based on current character classifier's recognition accuracy (approx. 89\%).

The missed segmentation points were due to the neural assistant misrecognising two joined characters as a single character. This type of error is very hard to deal with, since when two characters are tightly coupled, the ligature cannot be detected. One solution is to employ a better neural classifier or incorporate more heuristic rules. However, in some cases the missed segmentations may be recovered when the neural-based segmentation technique is employed, which uses the centre area associated with each segmentation point. Figure 5 provides some sample handwriting with segmentation points found by EHS.

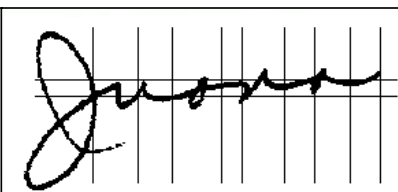

(a)

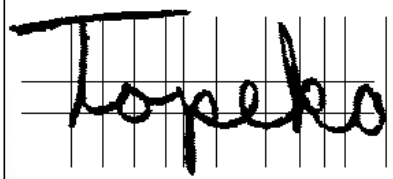

(c)

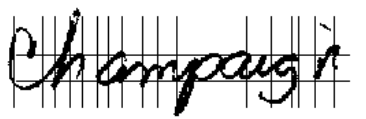

(e)

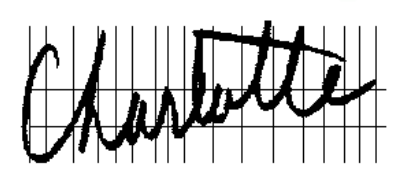

(b)

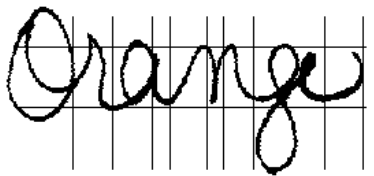

(d)

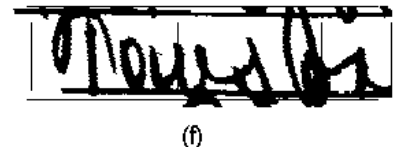

(f)
Figure 5: Sample word images segmented by the enhanced feature-based heuristic segmenter.

(a), (b), (c) successful words. (d), (e), (f) unsuccessful words.

Although neural classifiers may contribute problems in some instances, their use in the described segmenter was very beneficial, because it could introduce more segmentation points without using complex heuristics.

\subsection{Analysis of Neural-based Segmentation}

As may be seen from Table 2, the segmentation technique was successful at discarding bad segmentation points as well as recovering "missed" segmentation points by adding them at large gaps between points in words based on the average character width. Both experiments recorded the same 'missed' error of only $0.1 \%$, which is a very promising result. Furthermore, the results also showed that the enhanced heuristic segmenter was able to produce better inputs to increase overall segmentation results.

The reason for the higher 'bad errors' by the neuralbased segmentation technique as compared to those obtained by the enhanced heuristic segmenter is because some 'missed errors' are turned into 'bad errors'. This is due to the technique recovering 'missed' segmentation points based on the average character width. In some cases, it could not perfectly locate the character boundary (using SPD) and hence contributed to the 'bad error'. Although the 'over-segmentation error' went up slightly 
as compared to previous work, it is possible to recover this at a later stage.

Another reason for the increase of the segmentation performance is related to the use of the MDF and the segmentation path-based character extraction technique (SPD). Since 'clean' characters can be extracted and MDF provides better features for the single classifier, the performance of LCV and CCV are improved.

\section{Conclusion and Future Work}

This paper describes an improved neural-based segmentation technique for cursive words. The technique included an enhanced heuristic segmenter to oversegment handwriting in addition to the use of an MDF extraction technique for SPV, LCV and CCV. The enhanced heuristic segmenter provided better inputs to the subsequent neural validation process. Encouraging results were obtained that can increase the over-all performance of a segmentation-based handwriting recognition system.

In the future, EHS will be used to locate prospective segmentation points from the training set facilitating retraining and testing of the SPV classifier for further enhanced performance. The above-mentioned technique will also be tested on a larger dataset to validate the improvements proposed. Finally, a new character extraction technique that uses the direction feature on the character's boundary will be investigated.

\section{References}

[1] R. G. Casey, "Moment Normalization of Handprinted Characters", IBM Journal of Research Development, 1970, pp. 548-557.

[2] M. Blumenstein, B. Verma and H. Basli, "A novel feature extraction technique for the recognition of segmented handwritten characters", Proceedings of the Seventh International Conference on Document Analysis and Recognition, (ICDAR '03), Edinburgh, Scotland, 2003, pp. 137141 .

[3] P. D. Gader, M. Mohamed and J-H. Chiang, "Handwritten Word Recognition with Character and Inter-Character Neural Networks", IEEE Transactions on Systems, Man, and Cybernetics-Part B: Cybernetics, 27, 1997, pp. 158-164.

[4] M. Blumenstein, X. Y. Liu and B. Verma, "A Modified Direction Feature for Cursive Character Recognition", Proceedings of the International Joint Conference on Neural Networks (IJCNN '04), Budapest, Hungary, 2004, pp. 29832987.

[5] B. Yanikoglu and P. A. Sandon, "Segmentation of Off-Line Cursive Handwriting using Linear Programming", Pattern Recognition, 31, 1998, pp. 1825-1833
[6] G. Nicchiotti and C. Scagliola, "A Simple and Effective Cursive Word Segmentation Method", Proceedings of the Seventh International Workshop on Frontiers in Handwriting Recognition, Amsterdam, 2000, pp. 499-504.

[7] X. Xiao and G. Leedham, "Knowledge-based Cursive Script Segmentation", Pattern Recognition Letters, 21, 2000, pp. 945954.

[8] M. Blumenstein, and B. K. Verma, "Analysis of Segmentation Performance on the CEDAR Benchmark Database", Proceedings of the International Conference on Document Analysis and Recognition (ICDAR '01), 2001, pp. 1142-46.

[9] M. Blumenstein and B. Verma, "A New Segmentation Algorithm for Handwritten Word Recognition", Proceedings of the International Joint Conference on Neural Networks (IJCNN '99), Washington D.C., 1999, pp. 878-882

[10] C. K. Cheng, X. Y. Liu, M. Blumenstein, and V. Muthukkumarasamy, "Enhancing Neural Confidence-Based Segmentation For Cursive Handwriting Recognition", 5th International Conference on Simulated Evolution And Learning (SEAL '04), Busan, Korea, 2004, SWA-8, CD-ROM Proceedings.

[11] C. K. Cheng, M. Blumenstein, "Improving the segmentation of Cursive Handwritten Words using Ligature Detection and Neural Validation", Asia Pacific International Symposium on Information Technology (APIS 2005), Gold Coast, Australia, 2005, pp 56-59.

[12] J. J. Hull, “A Database for Handwritten Text Recognition”, IEEE Transactions of Pattern Analysis and Machine Intelligence, 16, 1994, pp. 550-554. 